\title{
Research in brief: Serum urate reduction and its effect on the progression of chronic kidney disease
}

\author{
Authors: Rajan S Pooni ${ }^{\mathrm{A}}$ and Richard Corbett ${ }^{\mathrm{B}}$
}

DOI: $10.7861 /$ clinmed.rib.20.5.1

\section{Background}

Summary of Doria A, Galecki AT, Spino C et al. Serum urate lowering with allopurinol and kidney function in type 1 diabetes. N Engl J Med 2020; 382:2493-503 and Badve SV, Pascoe EM, Tiku A et al. Effects of allopurinol on the progression of chronic kidney disease N Engl J Med 2020; 382:2504-13.

The worldwide incidence of chronic kidney disease (CKD) is rising; the burden within the UK is already high, with an estimated prevalence of $2.7 \%{ }^{1,2} \mathrm{CKD}$ is not a single disease but the consequence of a number of different pathologies resulting in progressive decline in kidney function, with implications for both cardiovascular health, along with a risk of developing end-stage renal failure.

Observational studies raised the possibility that reducing serum urate could halt the progression of CKD. ${ }^{3}$ Higher serum urate levels have been observed in patients with CKD compared with those without. ${ }^{4,5}$ More specifically, high serum urate is a strong independent predictor of decline in glomerular filtrate rate (GFR) and albuminuria in people with type 1 diabetes mellitus (T1DM). ${ }^{6}$ Additionally, a direct correlation has been observed between serum urate levels and cardiovascular morbidity and mortality in this population. ${ }^{7}$

Allopurinol, given its low cost, safety profile and wide availability, is therefore an attractive medication to potentially stem the progression of CKD; a concept supported by observational studies and small clinical trials. ${ }^{8-10}$ We review two recent and significant multi-centre randomised controlled trials (RCTs) examining the efficacy of serum urate reduction with allopurinol on the progression of mild-to-moderate CKD over a period of up to 3 years.

\section{Randomised controlled trial data}

The first RCT, which recruited people with T1DM and evidence of diabetic kidney disease (GFR $>40 \mathrm{~mL} / \mathrm{min} / 1.73 \mathrm{~m}^{2}$ ), enrolled 530 patients; 267 patients received allopurinol and 263 patients received a placebo. The primary outcome was iohexol-determined GFR after 3 years plus a 2-month washout period. Secondary outcome measures included GFR at specified intervals, urinary albumin excretion rate and incidence of cardiovascular events (both fatal and non-fatal).

The second RCT recruited 369 patients with stage 3 or 4 CKD (eGFR of $15-60 \mathrm{~mL} / \mathrm{min} / 1.73 \mathrm{~m}^{2}$ ) and significant albuminuria; 185 were assigned allopurinol and 184 assigned a placebo, with follow-up through to 2 years. Similarly, the primary outcome was change in GFR from baseline, determined by the creatinine-based CKD Epidemiology Collaboration (CKD-EPI) equation. Selected secondary outcome measures included incidence of end-stage renal failure, cardiovascular events and all-cause mortality.

Despite adequate powering and effective urate lowering in the interventional arms, neither study could demonstrate a change in GFR in mild-to-moderate CKD when compared to the placebo population (intention-to-treat analysis). Furthermore, incidence of cardiovascular events and all-cause mortality was similar in both groups across both RCTs.

Authors: ${ }^{A}$ internal medicine trainee, Newham University Hospital, Barts Health NHS Trust, London, UK; ${ }^{B}$ consultant nephrologist, Hammersmith Hospital, Imperial College Healthcare NHS Trust, London, UK
While these studies excluded younger people with early renal impairment who may benefit, the findings are in keeping with a trial of febuxostat, a non-purine inhibitor of xanthine oxidase, which also failed to demonstrate improved outcomes in the interventional arm. ${ }^{11}$ A large trans-ancestry genome-wide association study has provided mechanistic insight into the findings from these trials. ${ }^{12}$ Transcriptional co-regulation appears to influence both genes involved in urate excretion as well as those associated with cardiometabolic traits. This would explain the clinical associations observed in individuals with higher serum urate levels but at the same time the failure of urate-lowering therapy to mitigate progression of CKD and cardiovascular disease.

> While population-based observational studies may show association, they are hypothesis-generating. Robust, multicentre, blinded RCTs are needed to define clinical efficacy even in the face of strong observational evidence.

> There is no clinically significant benefit in serum urate reduction with allopurinol on kidney function or cardiovascular events in people with mild-to-moderate CKD.

> The apparent association between serum urate and CKD may be due to underlying common genetic factors. Transcriptional co-regulation results in the observed association between serum urate and cardiometabolic traits, with the latter particularly implicated in the progression of CKD. .

\section{References}

1 Hamer RA, El Nahas AM. The burden of chronic kidney disease. BMJ 2006;332:563-4.

2 Insight Health Economics. Chronic kidney disease in England: The human and financial cost. NHS Kidney Care, 2012.

3 Jalal DI, Chonchol M, Chen W, Targher G. Uric acid as a target of therapy in CKD. Am J Kidney Dis 2013;61:134-46.

4 Weiner DE, Tighiouart $\mathrm{H}$, Elsayed EF et al. Uric acid and incident kidney disease in the community. J Am Soc Nephrol 2008;19:1204-11.

5 Mauer M, Doria A. Uric acid and diabetic nephropathy risk. Contrib Nephrol 2018;192:103-9.

6 Afkarian M, Polsky S, Parsa A et al. Preventing Early Renal Loss in Diabetes (PERL) Study: A Randomized double-blinded trial of allopurinol-rationale, design, and baseline data. Diabetes Care 2019;42:1454-63.

7 Pilemann-Lyberg S, Hansen TW, Tofte $\mathrm{N}$ et al. Uric acid is an independent risk factor for decline in kidney function, cardiovascular events, and mortality in patients with type 1 diabetes. Diabetes Care 2019;42:1088-94.

8 Goicoechea M, de Vinuesa SG, Verdalles U et al. Effect of allopurinol in chronic kidney disease progression and cardiovascular risk. Clin J Am Soc Nephrol 2010;5:1388-93.

9 Goicoechea M, de Vinuesa SG, Verdalles U et al. Allopurinol and progression of CKD and cardiovascular events: long-term follow-up of a randomized clinical trial. Am J Kidney Dis 2015;65:543-9.

10 Siu YP, Leung KT, Tong MK, Kwan TH. Use of allopurinol in slowing the progression of renal disease through its ability to lower serum uric acid level. Am J Kidney Dis 2006;47:51-9.

11 Kimura K, Hosoya T, Uchida S et al. Febuxostat therapy for patients with stage 3 CKD and asymptomatic hyperuricemia: A randomized trial. Am J Kidney Dis 2018;72:798-810.

12 Tin A, Marten J, Halperin Kuhns VL et al. Target genes, variants, tissues and transcriptional pathways influencing human serum urate levels. Nat Genet 2019;51:1459-74. 